\title{
Analysis of emulsions concentration changes during imbibition in porous sorbents
}

\author{
O. S. Shtyka ${ }^{1}$ M. M. Błaszczyk ${ }^{1}$ J. P. Sęk ${ }^{1}$
}

Received: 5 April 2016/Revised: 24 June 2016/Accepted: 19 July 2016/Published online: 10 August 2016

(C) The Author(s) 2016. This article is published with open access at Springerlink.com

\begin{abstract}
The stabilized emulsions removal from the aqueous systems is supposed to be an issue as important as the free oil products recovery. This work was conducted to investigate the mechanisms of oil-in-water emulsions sorption in a non-woven oleophilic/hydrophobic material forced by the capillary suction pressure, and the saturation level of such material during this process. It ensures the effective application of the sorbents and facilitates the development of alternative high-performance sorptive materials that can contribute to diminishing of the negative consequences of the aquatic environment contamination. The main aspect investigated in this research work was kinetics of the imbibition process in the porous media to define the optimal time of sorption and the height of the emulsions penetration. The measurement of the dispersed phase concentration changes with the increase in an emulsion front in the porous medium using the nephelometrical method is the advanced approach in the field of assessment of sorbents efficiency. The tendency of the imbibed emulsion concentration decrease versus the height was observed, which depended strongly on the viscosity and the initial composition of a penetrating liquid. The observed process was described by the mathematical model based on Hill equation.
\end{abstract}

Keywords Dispersed phase concentration - Emulsion . Hill equation · Imbibition · Porous sorbent $\cdot$ Saturation

O. S. Shtyka

olga.shtyka@edu.p.lodz.pl

1 Department of Chemical Engineering, Faculty of Process and Environmental Engineering, Lodz University of Technology, Wolczanska 213, 90-924 Lodz, Poland

\author{
Abbreviations \\ API American Petroleum Institute \\ DPC Dispersed phase concentrations \\ KO Kerosene oil \\ VO Vegetable oil
}

\section{Introduction}

Over the last decades, the contamination of the aqueous environment with oil and its derivative compounds has been recognized as a serious ecological problem. Such contaminants can be present in aqueous objects in the form of free spilled oil products or their emulsions. In the majority of cases, the penetration of different types of emulsions is a direct result of the surface run-off from the land-based objects and the discharge of industrial untreated effluents, i.e. from oil refinery facilities, objects of food industry, metal processing, refuelling and service stations, territories with storage tanks, and also during transportation operations (Wake 2005; Srinivasan and Viraraghavan 2010; Coca-Prados and Gutiérrez-Cervelló 2011). The reason of such contamination can also be oil spills caused mainly as a result of drilling processes, transportation, e.g. leakages from tankers, underwater and underground pipelines, and accidents on offshore platforms (Fingas 2011; Al-Majed et al. 2012). Following the oil and/or its refined products penetration into the aquatic environment, the sequence of physical, chemical, and biological weathering processes and contaminants translocation (i.e. spearing, drifting) occurs. The climatic and environmental conditions as well as the properties of oil-spill-derived hydrocarbons are recognized to be the dominating factors 
that define the kinetics and mechanisms of the oil transformation, including the emulsification and dispersion processes (Daling and StrØm 1999; Kingston 2002; Fingas 2011).

One of the initially required conditions for the abovementioned alterations of thin layer of oil on water surface is a wind speed in a range $3-5 \mathrm{~m} / \mathrm{s}$. However, emulsification and dispersion of the spilled oil can also be intensified by a mixing intensity of waves and streams (Wei et al. 2003; AlMajed et al. 2012). The oil-in-water dispersions and waterin-oil emulsions are reported to be formed on the surface and at near surface zone of a water column (Walker et al. 1993; Lewis et al. 1994; Xie et al. 2007).

The main consequences of such a contamination are economic losses and a wide range of negative impacts on the environment. Subject to the oily component properties of an emulsion, the threats can be represented by toxic, carcinogenic, and mutagenic effects on aquatic and coastalterrestrial biota, an inhibition of the physiological activity of living organisms, a disruption of the food chains, and an irreversible degradation of the ecosystem due to the deterioration of its abiotic components quality (Kingston 2002; Wake 2005; Fingas 2011).

Presently, a great deal of different remediation approaches was elaborated for oil and its products removal from the aqueous media, which application depends on the scale, the character of the contaminated territory, and the spilled pollutants properties. Emulsions recovery process can be based on the technologies such as coagulation and flocculation, gravity separation, chemical treatment, dissolved air flotation, membrane processes, and integrated or hybrid methods (Fakhru'l-Razi et al. 2009). However, all of them feature some major disadvantages such as a low efficiency in small oil droplets removing, a high operation costs, and some difficulties in applying in situ (Fakhru'l-Razi et al. 2009; Coca-Prados and Gutiérrez-Cervelló 2011). In contrast, the mechanical extraction by use of suitable sorbents can be considered as one of the most effective method for emulsions removal due to its ease in handling and implementing in the contaminated areas. Furthermore, the mechanical recovery using sorptive materials is also regarded as a highly appropriate countermeasure due to the absence of risk concerning an additional environmental contamination.

Among different types of sorbents, i.e. natural, inorganic mineral, and synthetic, preferably used are the last ones because of a high-efficiency and a low-cost technology as well as numerous exploitation advantages. The results of researches related to the effectiveness of polypropylene, polyethylene, polystyrene and their modified variants as sorbents of oils, and its refined products from water surface were discussed in the scientific literature (Teas et al. 2001; Wei et al. 2003; Wei et al. 2005; Zhu et al. 2011; Lin et al. 2012; Wu et al. 2012).

Most of commercial polypropylene sorbents are in the form of non-woven materials that consist of webs created by consolidation of individual fibres. Such a structure provides a formation of voids that facilitates the penetration of a contaminant and its retention into the sorbents (Schmenk et al. 2000). Teas et al. compared the oil-sorption capacity of polypropylene with commercial types of perlite and cellulosic material. The synthetic material exhibited a high-performance absorption of light cycle and light gas oils in all sets of experiments in both cases of pure compounds and oils presence in water baths (Teas et al. 2001). Different samples of polypropylene non-woven materials were tested according to their initial oil-sorption capacities and oil-retention properties in another publication (Wei et al. 2003). Using the wasted plastic in the form of powders and sheets as a sorbent of oil products was also investigated. The polyethylene powder and sheets as well as polypropylene powder were recommended for heavy crude oils removing, while polypropylene sheets adsorbed light oils (Aboul-Gheit et al. 2006).

A part of the literature is also dedicated to the methods of synthetic sorbents modification and estimation of their abilities to be applied for the oil-spill-derivative hydrocarbons removal (Ceylan et al. 2009; Qi et al. 2011; Zhu et al. 2011; Lin et al. 2012; Atta et al. 2013; Saleem et al. 2014). The efficiency of the macroporous polymeric material consisting of butyl rubber as a sorbent was investigated in laboratory conditions. Its sorption capacity for crude oil and petroleum products was defined in a range of $15-23 \mathrm{~g} / \mathrm{g}$ (Ceylan et al. 2009). The commercial felt was modified by adding expanded graphite with an oil-sorption capacity of $80 \mathrm{~g} / \mathrm{g}$. The experimental results indicated that the absorption capacity of the obtained sample was remarkably improved, i.e. almost by $300 \%$ in comparison with the application of the pure felt (Qi et al. 2011). The sorption capacity of a sorbent consisting of polyvinyl chloride (PVC) and polystyrene (PS) fibre prepared by an electrospinning process was investigated in the publication of Zhu et al. (2011). The results showed that the sorption ability of the PVC/PS sorbent for different oily liquids can be displayed in such order: motor oil $>$ peanut oil $>$ ethylene glycol $>$ diesel (Zhu et al. 2011). Polystyrene produced by means of the electrospinning method and characterized with high specific surface of fibres was reported to be an excellent sorbent for vegetable and motor oils due to the combination of absorption and adsorption 
processes (Lin et al. 2012). On the other hand, reutilization of such sorbent and extraction of the imbibed liquid from fibres pores can become a quite challenging and complicated process. The efficiency of non-woven polyethylene terephthalate fibres modified by the adherence of crosslinked polymer coatings to the fibre surface was evaluated by means of oil absorption experiments with toluene and crude oil as the sorbed media (Atta et al. 2013). More recently, the diesel oil uptake process by a synthetic sorbent consisting of ultra-high molecular weight polyethylene has also received some attention (Saleem et al. 2014). Despites the high sorption capacity, the modified polymer sorbents have the following drawbacks: high production costs of these materials, fabrication mainly in laboratory scale, and finally, a low reusability level of multicomponent sorbents and these composed of nanoporous fibres.

The sorption process is based on the physical phenomenon known as the spontaneous imbibition forced by capillary rise. The capillary pressure is the main acting force of the imbibition, and it appears due to the wetting of the porous medium by a penetrating liquid, and consequent interactions of their surface energies (Zhmud et al. 2000; Hamraoui and Nylander 2002; Masoodi et al. 2007). There has been an ongoing interest in the imbibition process carried out by science and industry (Zhmud et al. 2000; Masoodi and Pillai 2010; Cai et al. 2012). The processes and mechanisms of porous media imbibition with singlephase liquids, for example, water (Hamraoui and Nylander 2002; Masoodi and Pillai 2010); deionized water and dimethyl silicone oil (Xue et al. 2006); dodecane and diethyl ether (Zhmud et al. 2000); and dodecane, hexadecane, and decane (Masoodi et al. 2007) were investigated experimentally and described in the literature. Moreover, the various models mainly based on fundamental equations such as Lucas-Washburn, Hagen-Poiseuille, and LaplaceYoung were proposed to approximate the experimental data concerning single-phase liquids imbibition in the capillaries and different porous media. Many of such models consider the influence of dynamic contact angle on the capillary rise (Zhmud et al. 2000; Hamraoui and Nylander 2002; Digilov 2008; Hilpert 2010). Another type related to the spontaneous imbibition of a wetting permeant into gas-saturated porous media consisting of a complex structure of pores and based on fractal geometry (Benavente et al. 2002; Zhao and $\mathrm{Li}$ 2009; Cai et al. 2010, 2011, 2012). The effect of tortuosity and shape on the capillary imbibition in the wetting porous media is discussed in the publications of Cai et al. (2010, 2011, 2012), and this approach allowed to characterize the mentioned process in a wide range of porous structures, i.e. rocks, silica glasses, and fibrous material.

The emulsions are recognized as the heterogeneous multiphase systems, consisting of immiscible components, which can differ at least by viscosity and wettability. This allowed to assume that the process of porous media imbibition with emulsions differed from the one with singlephase liquids. Moreover, the previously mentioned models were applicable to describe transport phenomenon driven by the capillary pressure in case of single-phase permeants and disregarded the effect of composition heterogeneity and the structural changes of multiphase penetrating liquids in porous media.

The experiments reported in the present paper were undertaken in order to study the changes of two-phase liquids behaviour during the porous oleophilic/hydrophobic medium imbibition. The porous structure saturation versus the height of an imbibed permeant penetration and the influence of the initial dispersed phase concentrations were the objective of the discussion. Moreover, the phenomenon of an imbibed emulsion concentration changes with the increase in its front height was an issue investigated in this publication. The observed process was described by the mathematical models based on the fundamental sorption equations. The experiments were performed during 2015-2016 at the Department of Chemical Engineering in Lodz University of Technology (Poland). In practice, this investigation aims at estimating the imbibition mechanism of synthetic sorbents to predict the process of different multiphase liquids removal from the aquatic environment.

\section{Materials and methods}

Six types of oil-in-water emulsions were prepared by means of a homogenizer MSM-67170 (Bosch, Germany) during $600 \mathrm{~s}$, which was sufficient to obtain the required degree of homogenization. According to the results of the microscopic images analysis (Microscope Leica DMI3000B with camera Lumenera Infinity1), the diameters of dispersed phase droplets in the obtained emulsions were in the range $1-20 \mu \mathrm{m}$, while $75-80 \%$ of them had the size of $2-5 \mu \mathrm{m}$. One of the experimental liquids was emulsions composed of kerosene Shellsol D-60 (ChemiaŁódź S.A., Poland) and denoted as KO type. They represented low-viscosity oil products such as diesel oil, light crude oil, and gasoline. Using kerosene was promoted by its relatively non-volatility that minimized physicochemical properties changes of medium during the experiments. 
Table 1 Properties of emulsions used in experiments $\left(T=23 \pm 1{ }^{\circ} \mathrm{C}\right)$

\begin{tabular}{lllll}
\hline DPC $(\%)$ & $\begin{array}{l}\text { Viscosity } \\
(\mathrm{mPa} \mathrm{s}) \\
\text { KO emulsion }\end{array}$ & $\begin{array}{l}\text { Density } \\
\left(\mathrm{kg} / \mathrm{m}^{3}\right)\end{array}$ & $\begin{array}{l}\text { Viscosity } \\
(\mathrm{mPa} \mathrm{s}) \\
\text { VO emulsion }\end{array}$ & $\begin{array}{l}\text { Density } \\
\left(\mathrm{kg} / \mathrm{m}^{3}\right)\end{array}$ \\
\hline 10 & $1.133 \pm 0.021$ & $973.1 \pm 2.15$ & $5.920 \pm 0.140$ & $992.2 \pm 2.31$ \\
30 & $2.220 \pm 0.050$ & $932.2 \pm 1.13$ & $14.850 \pm 0.23$ & $978.9 \pm 3.43$ \\
50 & $5.120 \pm 0.003$ & $890.8 \pm 1.38$ & $28.930 \pm 0.24$ & $961.9 \pm 2.18$ \\
\hline
\end{tabular}

The kerosene API density was 52.74 $(\rho=767.80 \pm$ $2.10 \mathrm{~kg} / \mathrm{m}^{3}$ ), and viscosity was $1.23 \pm 0.20 \mathrm{mPa}$ s measured at $23{ }^{\circ} \mathrm{C}$. The value of surface tension was $27 \pm 1.8 \mathrm{mN} / \mathrm{m}$. The emulsions of VO type consisted of the refined vegetable oil Uniwersalny (FHPU Marlibo Z.P.Chr., Poland), which served as a model liquid of highviscous oil products. Its API density was equal to $21.97^{\circ}$, and density was equal to $922.00 \pm 0.60 \mathrm{~kg} / \mathrm{m}^{3}$. The viscosity value for oil was $53.12 \pm 0.44 \mathrm{mPa} \mathrm{s}$, and surface tension was $32 \pm 1.7 \mathrm{mN} / \mathrm{m}$.

The dispersed phase concentrations (DPC) of the prepared emulsions were 10,30 , and 50 vol\%. The emulsions were stabilized with a non-ionic surfactant-ethoxylated oleic acid (commercial name Rokacet O7, produced by PCC Exol SA, Poland). It was added in an amount of 2 per $100 \mathrm{ml}$ of an emulsion.

The viscosity of the emulsions and the basic components was measured using a shear rheometer Bohlin CVO120 (Malvern Instruments, UK). The density was determined by means of the picnometric method. Three replications were carried out for each measured parameter of an emulsion, and results were presented as their mean values. The physicochemical properties of the prepared liquid media are shown in Table 1 .

A commercial non-woven polypropylene material (Sintac (C-Polska Sp.z o.o., Poland) with an average porosity equal to $0.93 \pm 0.01$ was used as the porous sorptive medium. This material is recognized as an appropriate sorbent for oil products removal from the environment. It revealed to have a sufficient sorption capacity of oily components of the investigated emulsions (i.e. $20 \mathrm{~g} / \mathrm{g}$ for the vegetable, $15 \mathrm{~g} / \mathrm{g}$ for kerosene oil), but it was not imbibed with water. The density of the investigated material was equal to $63.84 \mathrm{~kg} / \mathrm{m}^{3}$. Such polypropylene sorbents are characterized by high level of physical and chemical resistance, reusability, and possibility to recover the absorbed oil. For the experimental purposes, the stripes were cut from a polypropylene sheet which had a width of $0.035 \mathrm{~m}$ and a length of $0.20 \mathrm{~m}$. The mean thickness of each stripe was equal to $0.0019 \mathrm{~m}$.

\section{Determination of the maximal mass and height of an imbibed liquid}

For the imbibition process investigation, the prepared emulsions were poured into a beaker and its initial mass $m_{\mathrm{o}}$ was measured using an analytical balance (Radwag WTB 200 , Poland) with an uncertainty of $\pm 0.001 \mathrm{~g}$. A stripe of polypropylene was directly immersed with one end into emulsion to a depth of $0.02 \mathrm{~m}$ (Sęk et al. 2015). The contact area of porous material with an emulsion was equal to $0.0014 \mathrm{~m}^{2}$. The changes of the emulsion mass in a beaker were registered as a function of time starting when the sorbent stripe was submerged and the process of imbibition initiated. The time when the mass of the emulsion in a beaker became steady was assumed as the final time of imbibition process $t_{\max }$. The achieved height of the liquid front at $t_{\max }$ was accordingly denoted as the equilibrium height, $h_{\max }$ (Sęk et al. 2015). Afterwards, the sample of sorbent imbibed with an emulsion was removed and the mass $m_{\mathrm{f}}$ of the emulsion left in the beaker was measured. The difference between the initial $m_{\mathrm{o}}$ and final mass was defined as the maximal imbibed mass: $m_{\max }=m_{\mathrm{o}}-m_{\mathrm{f}}$. For each type of emulsion, at least three independent experiments were conducted and the mean values were calculated. All experiments were conducted at $23 \pm 1{ }^{\circ} \mathrm{C}$.

\section{Determination of an emulsion concentration changes during sorption process}

These experiments allowed to define the variation of an emulsion concentration with its front raise in the porous media during the imbibition and to evaluate the influence of the following factors: initial dispersed phase concentration and properties of the oil used as an internal phase in the emulsions. The experiments were conducted for the maximal time of process $t_{\max }$, which in this case was equal to $1800 \mathrm{~s}$, and also for shorter intervals $t_{\text {im }}$ such as 300 , 900 , and $1200 \mathrm{~s}$. There was no considerable difference between the results for 1200 and 1800 s; therefore, only the 


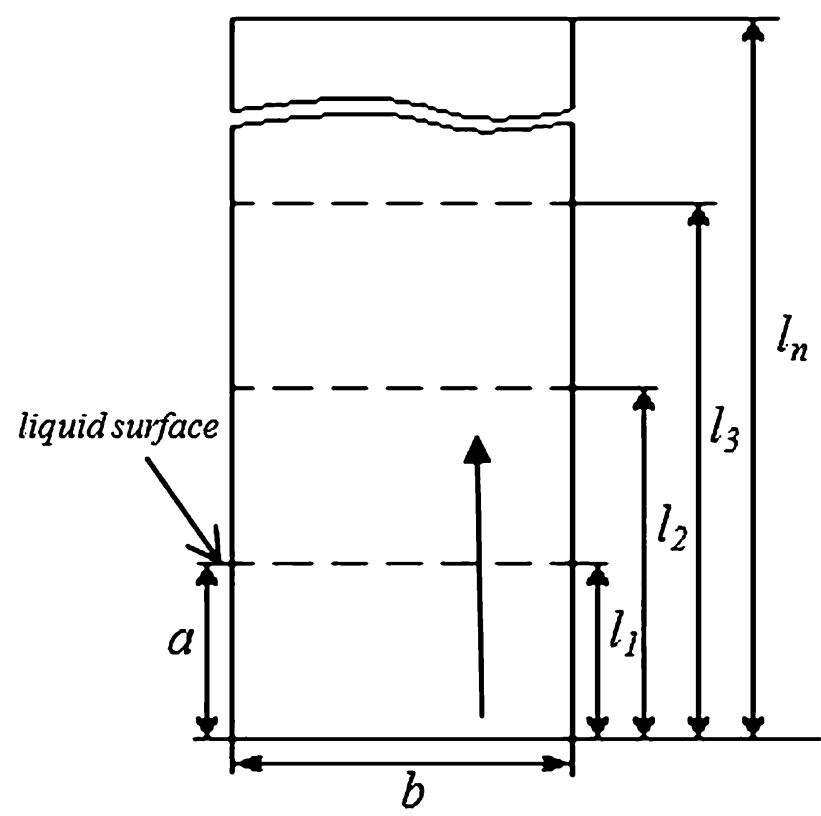

Fig. 1 Scheme of the sorbent sample division: $a$ the height and $b$ the width of a sorbent fragment

last data point was used for plotting the results. After such a defined time, a sorbent stripe was removed from the beaker, and its soaked part was cut into fragments. The principle of a sorbent division is shown in Fig. 1.

Each fragment had identical dimensions, i.e. the height $a$ of $0.020 \mathrm{~m}$ and the width $b$ of $0.035 \mathrm{~m}$. The first fragment $L_{1}$ visualized a submerged part of material, and its height range was from 0 to $0.02 \mathrm{~m}$. The height boundaries of next fragments were calculated according to the formula: $L_{n}=l_{n}-l_{n-1}$; thus, $L_{2}$ represented the height in the range $0.02-0.04 \mathrm{~m}$, for $L_{3}$, it was equal to $0.04-0.06 \mathrm{~m}$ etc. (Fig. 1).

The samples of material were washed out after imbibition with $10 \mathrm{ml}$ of distillated water. After that, the mass of the washed out liquid and a part of retained liquid in the material fragment were measured. The nephelometrical method was applied to determine concentrations of the dispersed phase in the obtained samples of liquids. The back-scattering rate (BSR) of light in an emulsion was measured by means of the optical analyzer TurbiscanTM LAB (Formulaction, France). The concentration of the dispersed phase in the obtained samples was determined using the calibration curves that describe the functional dependence between BSR of the light in an emulsion and its concentration, as described elsewhere (Lemarchand et al. 2003).

\section{Results and discussion}

\section{Changes of the height of an emulsion front versus time}

The height of an imbibed emulsion front is an important parameter that characterizes the level of penetration inside the porous sorbent defining the effectiveness of the used material. The results of height changes with time are shown in Table 2.

These results clearly show that for time $300 \mathrm{~s}$, the highest levels of penetration were observed for $50 \%$ emulsions, i.e. KO-50 - $0.045 \mathrm{~m}$; VO-50 - $0.042 \mathrm{~m}$, in comparison with others, while the lowest heights of the emulsions front were in the case of VO-10 and KO-10 (Table 2).

For $t_{\mathrm{im}}=900 \mathrm{~s}$, the height of the front in porous medium was maximum for emulsions with lower dispersed phase concentrations, viz. KO- $10-0.098 \mathrm{~m}$ and VO-10 - $0.066 \mathrm{~m}$ (Table 2). The same tendency was noticed for the maximal time $t_{\max }$. Thus, for $10 \%$ emulsions, the height values were in the range $0.120-0.122 \mathrm{~m}$, whereas for others, it was lower, i.e. $0.102-0.105 \mathrm{~m}$ for $30 \%$ and $0.08 \mathrm{~m}$ for $50 \%$ emulsions (Table 2).

In summary, the height of imbibed emulsion front in the porous sorbent decreased with increasing the initial concentration of the dispersed phase at time $t_{\mathrm{im}} \geq 900 \mathrm{~s}$. The observed tendency can be explained by oleophilic/hydrophobic characteristics of the investigated porous sorbent. Consequently, emulsions with higher content of oleic substances as the dispersed phase would be absorbed higher level during first $300 \mathrm{~s}$. After that, the imbibition process began to slow down possibly due to the penetration

Table 2 Maximal height of the investigated emulsions penetration in the porous sorbent, $[\mathrm{m}]$

\begin{tabular}{lllllll}
\hline Time (s) & \multicolumn{6}{l}{ Type of imbibed emulsion } \\
\cline { 2 - 7 } & VO-10 & VO-30 & VO-50 & KO-10 & KO-30 & KO-50 \\
\hline 300 & 0.030 & 0.041 & 0.042 & 0.042 & 0.043 & 0.045 \\
900 & 0.066 & 0.064 & 0.062 & 0.098 & 0.096 & 0.075 \\
1800 & 0.120 & 0.102 & 0.080 & 0.122 & 0.105 & 0.080 \\
\hline
\end{tabular}


paths blocking with oil droplets or/and the increase in the balancing force. In case of $10 \%$ emulsions, oil is present in a low concentration and wet pores walls creating path for continuous phase that can explain lower height of penetration. Thereafter, these emulsions can penetrate at higher levels due to their low viscosity in comparison with $50 \%$ emulsions and possibly, the absence of significant paths obstruction with oil droplets.

\section{Saturation of the porous sorbent with imbibed emulsions}

The saturation characterizes the sorbent efficiency and defines a level of free volume filling. The porous sorbent saturation was calculated according to the following equation:

$S_{\mathrm{f}}=\frac{\left(V_{\mathrm{w}}+V_{\mathrm{r}}\right)-V_{\mathrm{s}}}{V_{\mathrm{f}} \varepsilon}$

where $S_{\mathrm{f}}$ is the degree of saturation in a fragment of a polypropylene stripe, [-]; $V_{\mathrm{w}}$ is the volume of an emulsion washed out from a fragment [ml]; $V_{\mathrm{r}}$ is the volume of a liquid retained in a fragment after washing out $[\mathrm{ml}] ; V_{\mathrm{s}}$ is the volume of the added water (in this case equal to $10 \mathrm{ml}$ ); $V_{\mathrm{f}}$ is the volume of the investigated fragment [ml]; and $\varepsilon$ is the porosity of a sorbent material.

The results of saturation changes versus the height for the equilibrium time $t_{\max }$ are represented in Fig. 2.

At $1800 \mathrm{~s}$, in the first fragment $\left(L_{1}\right)$, the volume of the imbibed and further extracted emulsions exceeded the potentially available free volume of porous medium in all investigated cases and sorbent was supposed to be oversaturated with the emulsions, i.e. $1 \leq S_{\mathrm{f} 1} \leq 1.4$ (Fig. 2).

This phenomenon can be explained by the fact that a part of emulsions was absorbed on the external surface creating a thin covering layer. In the second fragment $\left(0.02<h_{\mathrm{im}} \leq 0.04 \mathrm{~m}\right)$, the saturation level was equal to 1.0 for VO-50 and 0.93 for KO-50 emulsion. However, for $30 \%$ emulsions, its values were in a range $0.86-0.87$, and finally, for $10 \%$, it was $0.76-0.77$ (Fig. 2). For a height of $0.08 \mathrm{~m}$, all kerosene-based emulsions nearly had the same saturation values, which were $0.33-0.36$. While for vegetable oil-based emulsions, the corresponding values are 21 - $27 \%$ higher and spanned in the range 0.40-0.46.

At height of $0.10 \mathrm{~m}$, the average value of saturation was equal to $0.20-0.25$ for all investigated liquids, besides $50 \%$ emulsions which permeated only to the height $\leq 0.08 \mathrm{~m}$ (Fig. 2; Table 2). Three emulsions, viz. KO-10, 30 and
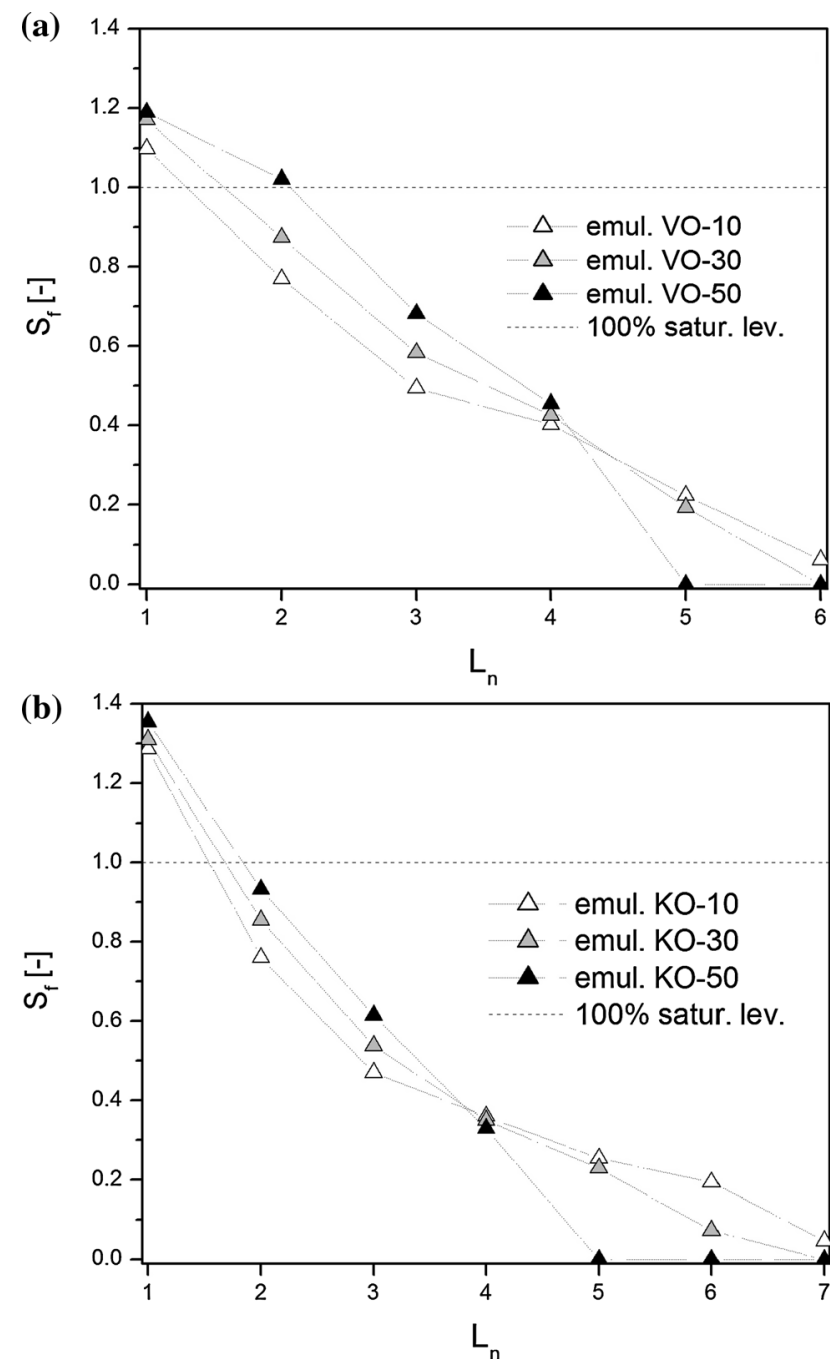

Fig. 2 Changes of saturation with the height of an imbibed emulsion front for $t_{\mathrm{im}}=1800 \mathrm{~s}$ : a for VO emulsion type; $\mathbf{b}$ for $\mathrm{KO}$ emulsion type

VO-10, reached the height $0.1-0.12 \mathrm{~m}$, and levels of porous material saturation were equal to $0.19,0.073$, and 0.062 , respectively.

As mentioned previously, only KO-10 achieved the highest value of maximal height in porous medium, i.e. $0.122 \mathrm{~m}$, but the observed sorbent saturation level was minimum in this case and was less than 0.05 (Fig. 2; Table 2).

Thus, these results suggest the following trends. Firstly, the sorbent saturation with an imbibed emulsion changes with the height of its front, and the minimal one was observed at the maximal achieved height. The analogous tendency was observed for all investigated emulsions. 

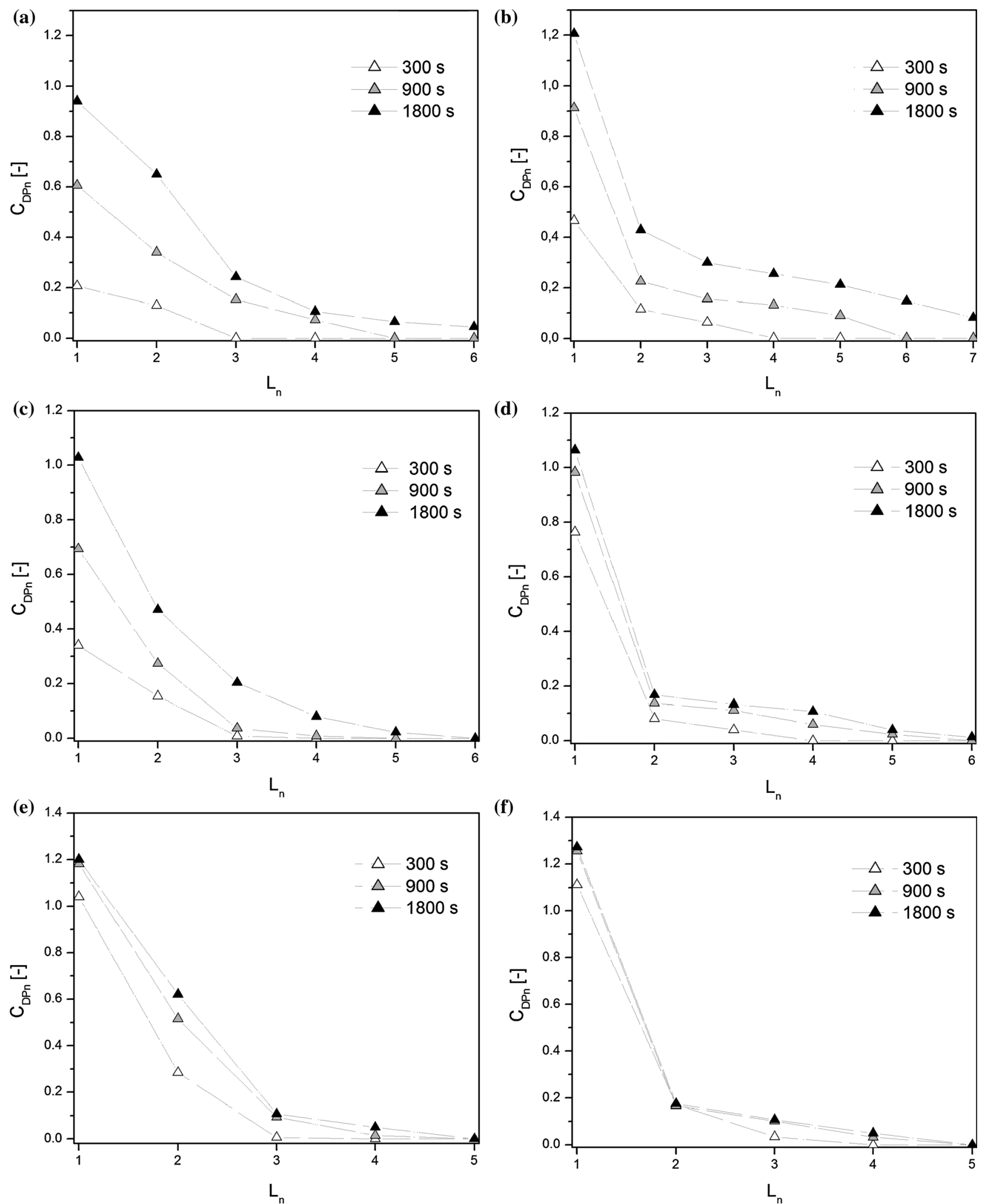

Fig. 3 Changes of the dispersed phase concentration versus the height at $t_{\mathrm{im}}=300,900$ and $1800 \mathrm{~s}$ for emulsions: a VO-10; b KO-10; $\mathbf{c}$ VO-30; d $\mathrm{KO}-30$; e VO-50; and f KO-50 
Secondly, the specific points were determined, for which saturation of polypropylene sorbent with the investigated emulsions was almost identical. Such point was defined at the height of $0.08 \mathrm{~m}$, and for $\mathrm{VO}$ emulsions, the average saturation level was equal to $\sim 0.43$, whereas for $\mathrm{KO}$ emulsions, it was approximately 0.35 (Fig. 2).

\section{Changes of the dispersed phase concentrations versus the height of emulsions penetration in a sorbent}

The emulsion concentration changes with the height for different times of the imbibition are shown in Fig. 3. The vertical axis on the graph represents the values of the normalized dispersed phase concentration which was denoted as $C_{\mathrm{DP} n}$ and calculated according to the expression:

$C_{\mathrm{DP}_{n}}=\frac{C_{\mathrm{DP}}}{C_{\mathrm{DP}_{0}}}$,

where $C_{\mathrm{DP}_{0}}$ is the initial concentration of the emulsion in a beaker before imbibition experiment and $C_{\mathrm{DP}}$ is the concentration of an imbibed emulsion at the defined height measured by the nephelometrical method.

The obtained concentrations of the dispersed phase were lower in the case of kerosene-based emulsions in comparison with another type (Fig. 3).

As shown in Fig. 3a, b, for $t_{\mathrm{im}}=1800 \mathrm{~s}$, the dispersed phase concentration in case of KO-10 was 2.4 times lower at the $h_{\mathrm{im}}=0.04 \mathrm{~m}$, than at the height of $0.02 \mathrm{~m}$, while for VO-10, this difference equalled 1.4 times. For emulsions with an initial concentration of $30 \%$, this difference was equal to 6.3 and 2.1 times for kerosene-based and vegetable oil-based emulsions, relatively (Fig. 3c, d). In the case of $50 \%$ emulsions, this value equalled 7.3 for $\mathrm{KO}$ type and 2.2 for VO type (Fig. 3e, f). Thus, the observations revealed that the process of an emulsion concentration decreasing is more gradual for vegetable oil-based emulsions rather than for kerosene-based ones.

Figures 4, 5 and 6 represent the results describing the difference between the changes of the dispersed phase concentration vs the height at the given value of time of the imbibition process. For the shortest investigated time, i.e. $300 \mathrm{~s}$, the highest emulsion concentrations were observed for both $50 \% \mathrm{VO}$ and $\mathrm{KO}$ emulsions (Fig. 4), whereas for $h_{\mathrm{im}} \leq 0.02 \mathrm{~m}$, the dimensionless dispersed phase concentrations differed significantly from each other. Thus, its values for vegetable oil-based emulsion were equal to 0.21 for $10 \%, 0.34$ for $30 \%$, and finally, 1.04 for $50 \%$ (Fig. 4a). In case of kerosene-based emulsion, the obtained
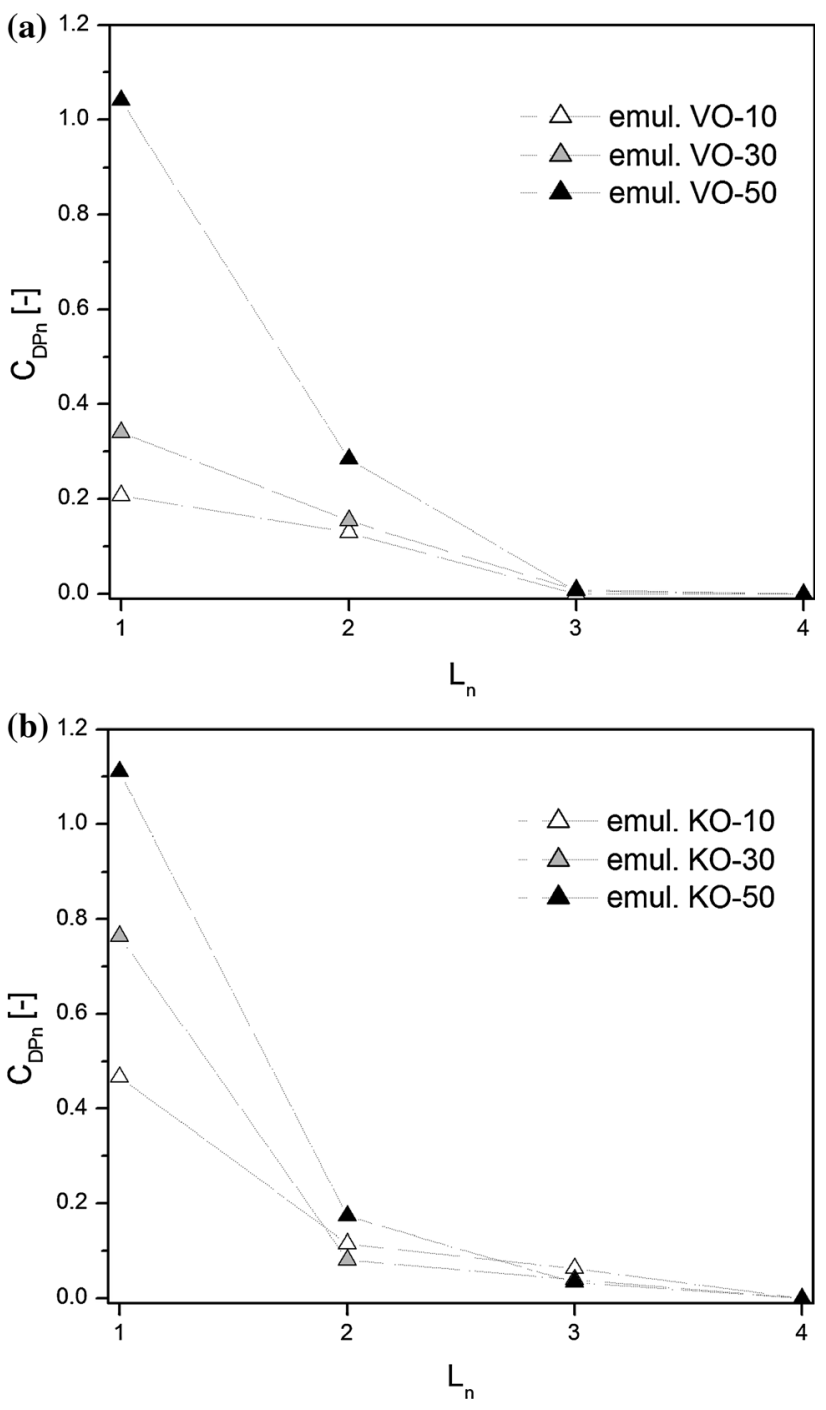

Fig. 4 Changes of the dispersed phase concentration with the height of an emulsion front for $t_{\mathrm{im}}=300 \mathrm{~s}$ : a for VO emulsions; $\mathbf{b}$ for $\mathrm{KO}$ emulsions

values of $C_{\mathrm{DP} n}$ at the same height were a little bit higher, i.e. 0.47 for $10 \%, 0.76$ for $20 \%$, and 1.11 for $50 \%$ (Fig. 4b). For $h_{\mathrm{im}}>0.04 \mathrm{~m}$, the rapid decreasing emulsion concentration was registered in this samples set. Moreover, the imbibed KO emulsions had almost identical concentrations of the dispersed phase for the height of $0.06 \mathrm{~m}$ (Fig. 4b).

As shown in Fig. 5a, b, the dispersed phase concentrations were much higher for time equal to $900 \mathrm{~s}$ in comparison with the results obtained for $300 \mathrm{~s}$. At the height of $0.06 \mathrm{~m}$ (i.e. $L_{3}$ ), the value of $C_{\mathrm{DP} n}$ for VO-10 equalled approximately 0.15 that was a higher concentration than in case of other emulsions, i.e. 0.037-0.094 (Fig. 5a). A 

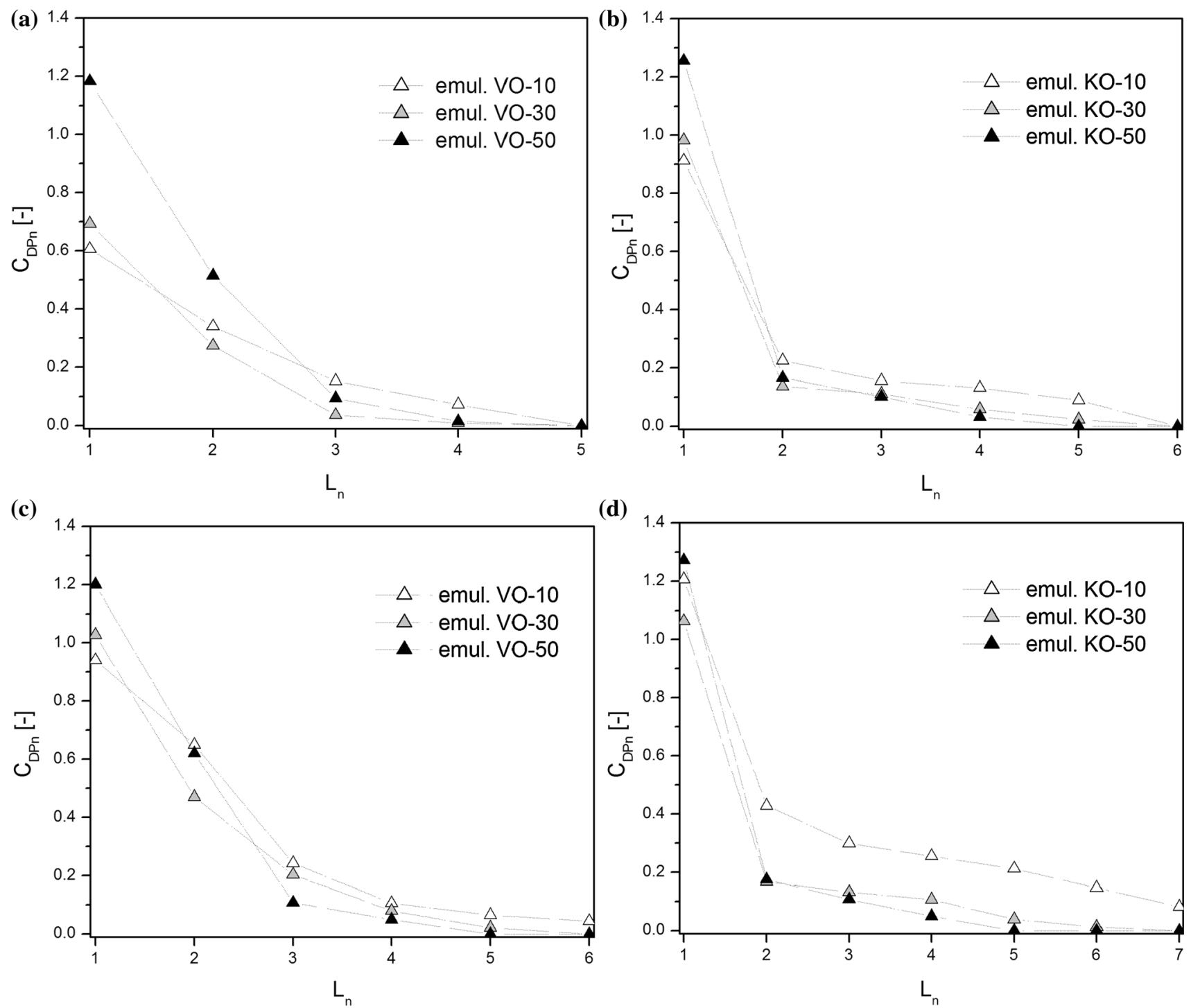

Fig. 5 Changes of the dispersed phase concentration with the height for: a VO emulsions, $t_{\mathrm{im}}=900 \mathrm{~s} ; \mathbf{b} \mathrm{KO}$ emulsions, $t_{\mathrm{im}}=900 \mathrm{~s} ; \mathbf{c} \mathrm{VO}$ emulsions, $t_{\mathrm{im}}=1800 \mathrm{~s}$; and $\mathbf{d} \mathrm{KO}$ emulsions, $t_{\mathrm{im}}=1800 \mathrm{~s}$

similar tendency was noticed for kerosene-based emulsions, but at $h_{\mathrm{im}} \leq 0.04 \mathrm{~m}$, viz. $L_{2}$ (Fig. $5 \mathrm{~b}$ ) and for all investigated emulsions in case of maximal imbibition time, i.e. $1800 \mathrm{~s}$ (Fig. 5c, d). The difference between concentrations for the same emulsions, but for process times of 900 and 1800 s, was relatively small (Fig. 5).

To sum up, the decrease in the dispersed phase concentration in relation to its initial value occurred slower for $10 \%$ emulsion than for other investigated liquids after the first $300 \mathrm{~s}$ of the imbibition time.

\section{Determination of the maximal mass of the imbibed emulsions}

The maximal mass of an imbibed emulsion $m_{\max }$ was measured experimentally and referred as a practically important parameter used to specify the effectiveness of the sorptive material. However, the obtained data concerning the saturation and the dispersed phase changes also allowed to calculate the maximal masses of imbibed liquids $M_{\max }$ in a sorbent and the following equation was used: 

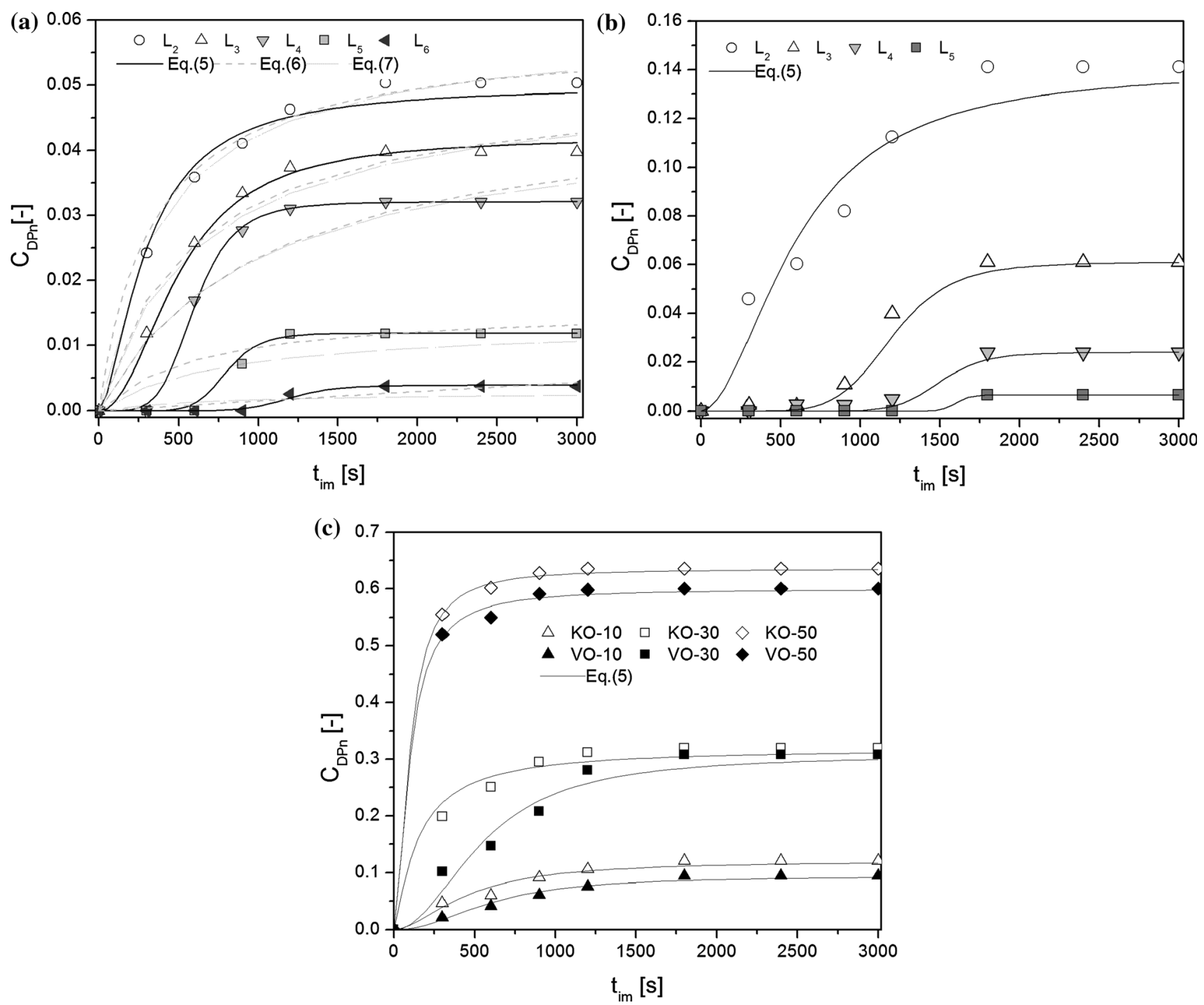

Fig. 6 Approximation of the experimental data for $t_{\mathrm{im}}=3000 \mathrm{~s}$ : a $30 \%$ kerosene oil-based emulsion; b $30 \%$ vegetable oil-based emulsion; and $\mathbf{c}$ all emulsions $\left(h_{\mathrm{im}} \leq 0.02 \mathrm{~m}\right)$

Table 3 Maximal masses of emulsions imbibed in the porous sorbent, ( $\mathrm{g}$ )

\begin{tabular}{lllllll}
\hline Method & \multicolumn{2}{l}{ Type of imbibed emulsion } \\
\cline { 2 - 6 } & VO-10 & VO-30 & VO-50 & KO-10 & KO-30 & KO-50 \\
\hline Experimental $\left(m_{\max }\right)$ & 4.15 & 4.38 & 4.47 & 4.53 & 4.44 & 4.15 \\
Analytical $\left(M_{\max }\right)$ & 4.23 & 4.49 & 4.56 & 4.62 & 4.53 & 4.20 \\
\hline
\end{tabular}

$M_{\max }=\left(V_{1} \varphi_{d 1} \rho_{d}+V_{1} \varphi_{c 1} \rho_{c}\right)+\cdots\left(V_{n} \varphi_{\mathrm{dn}} \rho_{d}+V_{n} \varphi_{\mathrm{cn}} \rho_{c}\right)$

where $V_{n}$ is the volume filled with an imbibed liquid in a fragment $n$ and defined as $S_{\mathrm{f}} V_{\mathrm{f}} ; \varphi_{\mathrm{dn}}$ is the fraction of the dispersed phase in a fragment $n ; \rho_{\mathrm{d}}$ is the density of the dispersed phase; $\varphi_{\mathrm{cn}}$ is the fraction of the continuous phase in a fragment $n$; and $\rho_{\mathrm{c}}$ is the density of the continuous phase.

The comparison of the maximal analytical mass and one obtained experimentally is represented in Table 3.

As shown in Table 3, the value of experimental mass differed slightly from calculated one, and the divergence was in a range $0.05-0.11 \mathrm{~g}$, i.e. $\sim 1.2-2.21 \%$. 


\section{Modeling of the dispersed phase concentration changes versus time}

The experimental data concerning the increase of an emulsion concentration versus time in the fragments of sorbent were approximated by equation based on fundamental descriptive-deterministic models such as Hill (Goutelle et al. 2008; Gesztelyi et al. 2012) and MichaelisMenten (Johnson and Goody 2011) relating the reaction velocity as a function of the substrate concentration, and Langmuir adsorption model (Zhang and Wang 2010). The models were chosen due to their mathematical form and common use in the fields of sorption processes description during which changes of a concentration occur.

The Hill equation is originally used to define the reactions kinetics and includes such parameters as the maximal reaction rate, $V_{\max }$; half-maximal concentration constant, $K$, and $n$ defined as the Hill coefficient. The classical form of equation is represented as follows:

$V=V_{\max } \frac{[S]^{n}}{K^{n}+[S]^{n}}$,

where $V$ is the rate of reaction per unit time, $S$ is the substrate concentration (Goutelle et al. 2008; Gesztelyi et al. 2012). In the presently discussed case, the parameter of the reaction rate was substituted by a dimensionless parameter of concentration $C_{\mathrm{DP} n}(t)$, and the substrate concentration, correspondingly by the time of an imbibition, $t_{\text {im }}$ [s]. Finally, after rearranging, one obtains such form of modified equation:

$C_{\mathrm{DP}_{n}}(t)=C_{\max } \frac{t_{\mathrm{im}}^{\Psi}}{t_{k}^{\Psi}+t_{\mathrm{im}}^{\Psi}}$,

where $C_{\max }$ is the dimensionless value of the maximal achieved concentration of oil phase in the fragment $L_{n}$ at the equilibrium time, $[-] ; t_{\mathrm{k}}$ is the time of an imbibition process, when the $C_{\mathrm{DP} n}$ is equal to a half of $C_{\max }$ [s]; and $\psi$ is defined as a coefficient of the given equation. Other proposed mathematical equations on the base of Langmuir and Michaelis-Menten models had the following form, respectively:

$C_{\mathrm{DP}_{n}}(t)=\alpha \frac{k \cdot t_{\mathrm{im}}}{1+k \cdot t_{\mathrm{im}}}$

$C_{\mathrm{DP}_{n}}(t)=C_{\max } \frac{t_{\mathrm{im}}}{\beta+t_{\mathrm{im}}}$

where $\alpha, k$ are defined as coefficients of Eq. 6 , and $\beta$ is a coefficient of the last equation.
The obtained results of the dispersed phase concentration changes are plotted as a function of the process time. One example of such plots is given in Fig. 6a and presents data for $30 \%$ kerosene oil-based emulsion and their approximation by the proposed Eqs. (5), (6), and (7). The dots depict the experimental results, while dashed lines show the values calculated by means of the proposed equations. The maximum observation time was assumed to be equal to $3000 \mathrm{~s}$; however, after the maximum sorption time of $1800 \mathrm{~s}$, only negligible changes of the emulsion front height were registered, i.e. $h_{\text {im }}<0.001 \mathrm{~m}$ per $300 \mathrm{~s}$. As shown in Fig. 6a, the most accurate fitting and highest accuracy was obtained in case of Eq. (5) appropriate for sigmoidal tendencies, which can describe the trend of the process at all investigated height of the permeant penetration in a sorbent. Considering this fact, only this mathematical equation was used to approximate the other experimental results. Other investigated liquids were characterized with the same high accuracy of fitting.

The analysis of a part of the sorbent samples, which were in contact with the emulsion allowed to define the common trends of the observed process for emulsions with the same initial concentration (Fig. 6c). The values of the parameters used for fitting with Eq. (5) are displayed in Table 4.

The adequacy of equation application for the experimental data fitting was estimated by means of the correlation coefficient, $R$, and the normalized root-meansquare error (NRMSE) (Gmurman 2004). The calculated values of the normalized root-mean-square error were in a range $0.013-0.085$ for all emulsions composed of vegetable oil and 0.031-0.086 for KO-10 and KO-30. However, the lowest one was obtained for $50 \%$ kerosene-based emulsion, i.e. 0.007-0.024. The values of the correlation coefficient were found to be in the range 0.950-0.999 for emulsion VO-10; 0.905-0.999 for VO30; 0.904-0.996 for VO-50; and 0.910-0.999 for emulsion KO-10; 0.977-0.998 for KO-30; 0.900-0.999 for KO-50. Therefore, the accuracy of the model can be accepted from the engineering point of view. Obviously, the values of parameters were different, whereas analogue tendencies in their changes were observed for both types of emulsions. Considering the results, one may assume that this equation gives a reasonable approximation of the experimental data because of the narrow boundaries within which solutions are found and adequate values of accuracy. 
Table 4 Parameters of the applied equation

\begin{tabular}{|c|c|c|c|c|c|c|c|c|c|}
\hline \multirow{3}{*}{$\begin{array}{l}\text { Fragment } \\
L_{1}\left(l_{1}\right)\end{array}$} & \multicolumn{9}{|c|}{ Parameters } \\
\hline & \multicolumn{3}{|c|}{ Emulsion VO-10 } & \multicolumn{3}{|c|}{ Emulsion VO-30 } & \multicolumn{3}{|c|}{ Emulsion VO-50 } \\
\hline & 0.094 & 600 & $2.16 \pm 0.26$ & 0.308 & 550 & $2.08 \pm 0.36$ & 0.600 & 100 & $1.64 \pm 0.09$ \\
\hline$L_{2}\left(l_{2}-l_{1}\right)$ & 0.065 & 750 & $2.65 \pm 0.49$ & 0.141 & 600 & $1.89 \pm 0.37$ & 0.310 & 350 & $1.83 \pm 0.26$ \\
\hline$L_{3}\left(l_{3}-l_{2}\right)$ & 0.024 & 800 & $5.73 \pm 0.71$ & 0.061 & 1200 & $6.52 \pm 1.73$ & 0.117 & 950 & $2.94 \pm 0.70$ \\
\hline$L_{4}\left(l_{4}-l_{3}\right)$ & 0.011 & 850 & $6.02 \pm 1.58$ & 0.024 & 1500 & $10.87 \pm 3.78$ & 0.070 & 1250 & $8.92 \pm 1.36$ \\
\hline$L_{5}\left(l_{5}-l_{4}\right)$ & 0.007 & 1100 & $9.42 \pm 2.89$ & 0.007 & 1600 & $27.55 \pm 3.53$ & - & - & - \\
\hline \multirow[t]{2}{*}{$L_{6}\left(l_{6}-l_{5}\right)$} & 0.005 & 1500 & $11.1 \pm 2.22$ & - & - & - & - & - & - \\
\hline & \multicolumn{3}{|c|}{ Emulsion KO-10 } & \multicolumn{3}{|c|}{ Emulsion KO-30 } & \multicolumn{3}{|c|}{ Emulsion $\mathrm{KO}-50$} \\
\hline$L_{1}\left(l_{1}\right)$ & 0.121 & 450 & $1.79 \pm 0.29$ & 0.319 & 150 & $1.32 \pm 0.13$ & 0.636 & 100 & $1.77 \pm 0.05$ \\
\hline$L_{2}\left(l_{2}-l_{1}\right)$ & 0.043 & 650 & $2.17 \pm 0.46$ & 0.050 & 300 & $1.61 \pm 0.15$ & 0.100 & 100 & $1.78 \pm 0.09$ \\
\hline$L_{3}\left(l_{3}-l_{2}\right)$ & 0.030 & 750 & $2.52 \pm 0.46$ & 0.040 & 500 & $2.61 \pm 0.29$ & 0.054 & 350 & $4.29 \pm 0.32$ \\
\hline$L_{4}\left(l_{4}-l_{3}\right)$ & 0.026 & 900 & $3.42 \pm 0.47$ & 0.032 & 600 & $4.89 \pm 0.35$ & 0.025 & 850 & $8.88 \pm 0.75$ \\
\hline$L_{5}\left(l_{5}-l_{4}\right)$ & 0.021 & 1200 & $4.38 \pm 1.25$ & 0.012 & 800 & $7.53 \pm 1.54$ & - & - & - \\
\hline$L_{6}\left(l_{6}-l_{5}\right)$ & 0.015 & 1400 & $9.74 \pm 0.82$ & 0.004 & 1200 & $8.50 \pm 3.34$ & - & - & - \\
\hline$L_{7}\left(l_{7}-l_{6}\right)$ & 0.008 & 1500 & $11.1 \pm 2.22$ & - & - & - & - & - & - \\
\hline
\end{tabular}

\section{Conclusion}

The process of porous sorbent imbibition with two-phase emulsions differs from that one with single-phase liquids. The height of an emulsion permeation in a porous medium forced by capillary pressure depends on an initial concentration of the dispersed phase. For example, the height in case of $10 \%$ emulsions was $\sim 18$ and $\sim 50 \%$ higher than for 30 and $50 \%$ emulsions relatively, when the process time was equal to $1800 \mathrm{~s}$. Thus, to remove emulsions with low concentrations, i.e. $\leq 10 \%$, applied sorbents should be a half higher in both cases of light and heavy oily components, than sorbent used for emulsions sorption with higher concentrations, i.e. $\geq 30 \%$.

The concentration of an emulsion during the imbibition process decreased with the increase in its front height in the porous sorbent in case of a non-immersed sorbent part, $h_{\mathrm{im}}>0.02 \mathrm{~m}$. Moreover, the kerosene-based emulsions were characterized by a quicker decreasing oil phase concentration with the height compared to another type of emulsions.

For a sorbent fragment with $h_{\mathrm{im}}<0.02 \mathrm{~m}$, an exceeding of its initial value of oil phase by $20-30 \%$ was observed for time of $1800 \mathrm{~s}$ in case of 10 and $30 \%$ emulsions, while for $50 \%$ emulsion, this phenomenon was registered even at minimal observation time, i.e. $t_{\mathrm{im}}=300 \mathrm{~s}$. Thus, it is possible to assume that droplets were retained in lower layers of the sorbent causing obstruction of the penetration paths.
The saturation degree is recognized as one of the substantial parameters to evaluate the efficiency of pores volume filling and as a consequence, the polypropylene sorbent application. The oversaturated zone was defined at $h_{\mathrm{im}}<0.02 \mathrm{~m}$ for all investigated emulsions. The observed exceeding of free pores volume of the material by $10-20 \%$ was explained by an extra layer formation on the sorbent external surface. In case of the non-submerged part, the maximal saturation was observed for a height value in range $0.02<h_{\mathrm{im}}<0.04 \mathrm{~m}$ (i.e. $L_{2}$ ). It was equal to 100 for $50 \%$ emulsions, while for $30 \%$, it was 86-87 \%, and near $77 \%$ for emulsions with the dispersed phase of $10 \%$. It proved once again that a larger part of the imbibed emulsions was retained in the lower part of a sorbent.

The experimental data concerning the dispersed phase concentration changes vs time for the different heights of an emulsion permeation in porous medium were approximated adequately by means of the modified Hill equation.

The present experimental results allow to define the appropriate time of sorption for non-modified polypropylene sorbent with an average porosity of $0.93 \pm 0.01$. Thus, its application time should not exceed $1800 \mathrm{~s}$, because after the equilibrium state of the system was observed that was proved by the unchangeable values of imbibed mass and height.

Acknowledgments The study was funded in framework of the Foundation Program for Young Scientists in Lodz University of Technology (Grant No. 501/10-34-2-1015) and Grand of Narodowy Centrum Nauki (No. 2013/05/N/ST8/03798). 


\section{Compliance with ethical standards}

Conflict of interest The authors declare that they have no conflict of interest.

Open Access This article is distributed under the terms of the Creative Commons Attribution 4.0 International License (http:// creativecommons.org/licenses/by/4.0/), which permits unrestricted use, distribution, and reproduction in any medium, provided you give appropriate credit to the original author(s) and the source, provide a link to the Creative Commons license, and indicate if changes were made.

\section{References}

Aboul-Gheit AK, Khalil FH, Abdel-Moghny T (2006) Adsorption of spilled oil from seawater by waste plastic. Oil Gas Sci Technol 61(2):259-268

Al-Majed AA, Adebayo AR, Hossain ME (2012) A sustainable approach to controlling oil spills. J Environ Manage 113:213-227

Atta AM, Brostow W, Hagg Lobland HE, Hasan ARM, Perezd JM (2013) Porous polymer oil sorbents based on pet fibers with crosslinked copolymer coatings. RSC Adv 3:25849-25857

Benavente D, Lock P, Cura M, Ordóñez S (2002) Predicting the capillary imbibition of porous rocks from microstructure. Transp Porous Med 49:59-76

Cai J, Yu B (2011) A discussion of the effect of tortuosity on the capillary imbibition in porous media. Transp Porous Med 89:251-263

Cai J, Yu B, Zou M, Luo L (2010) Fractal characterization of spontaneous co-current imbibition in porous media. Energy Fuels 24:1860-1867

Cai J, Hu X, Standnes DS, You L (2012) An analytical model for spontaneous imbibition in fractal porous media including gravity. Colloids Surf A 414:228-233

Ceylan D, Dogu S, Karacik B, Yakan SD, Okay OS, Okay O (2009) Evaluation of butyl rubber as sorbent material for the removal of oil and polycyclic aromatic hydrocarbons from seawater. Environ Sci Technol 43(10):3846-3852

Coca-Prados J, Gutiérrez-Cervelló G (2011) Water Purification and Management, 1st edn. Springer, Netherlands. doi:10.1007/97890-481-9775-0

Daling PS, StrØm T (1999) Weathering of oils at sea: model/field data comparisons. Spill Sci Technol Bull 5(1):63-74

Digilov RM (2008) Capillary rise of a non-Newtonian power law liquid: impact of the fluid rheology and dynamic contact angle. Langmuir 24(23):13663-13667

Fakhru'l-Razi A, Alireza P, Luqman CA, Dayang Radiah AB, Sayed SM, Zurina Zainal A (2009) Review of technologies for oil and gas produced water treatment. J Hazard Mater 170:530-551

Fingas M (2011) Oil spill science and technology: prevention, response, and clean up, 1st edn. Elsevier, Burlington, pp 7-1117-39

Gesztelyi R, Zsuga J, Kemeny-Beke A, Varga B, Juhasz B, Tosaki A (2012) The Hill equation and the origin of quantitative pharmacology. Arch Hist Exact Sci 66(4):427-438

Gmurman VE (2004) Probability theory and mathematical statistics, 10th edn. Higher School, Moscow (in Russian)

Goutelle S, Maurin M, Rougier F, Barbaut X, Bourguignon L, Ducher M, Maire P (2008) The Hill equation: a review of its capabilities in pharmacological modelling. Fundam Clin Pharmacol 22(6):633-648
Hamraoui A, Nylander T (2002) Analytical approach for the LucasWashburn equation. J Colloid Interface Sci 250(2):415-421

Hilpert M (2010) Liquid withdrawal from capillary tubes: explicit and implicit analytical solution for constant and dynamic contact angle. J Colloid Interface Sci 351(1):267-276

Johnson KA, Goody RS (2011) The original Michaelis constant: translation of the 1913 Michaelis-Menten paper. Biochemistry 50(39):8264-8269

Kingston P (2002) Long-term environmental impact of oil spills. Spill Sci Technol Bull 7(1-2):53-61

Lemarchand C, Couvreur P, Vauthier C, Costantini D, Gref R (2003) Study of emulsion stabilization by graft copolymers using optical analyzer Turbiscan. Int J Pharm 254:77-82

Lewis A, Daling PS, Strom-Kristiansen T, Singsaas I, Fiocco RJ, Nordvik AB (1994) Chemical dispersion of oil and water-in-oil emulsions-a comparison of bench scale test methods and dispersant treatment in meso-scale flume. In: Proceedings of the seventeenth arctic and marine oil spill program. Ottawa, Canada, pp 979-1010

Lin J, Shang Y, Ding B, Yan J, Yu J, Al-Deyab SS (2012) Nanoporous polystyrene fibers for oil spill cleanup. Mar Pollut Bull 64(2):347-352

Masoodi R, Pillai KM (2010) Darcy's law-based model for wicking in paper-like swelling porous media. AlChE J 56(9):2257-2267

Masoodi R, Pillai KM, Varanasi PP (2007) Darcy's law based models for liquid absorption in polymer wicks. AlChE J 53(11):2769-2782

Qi X, Jia Z, Yang Y, Liu H (2011) Sorption capacity of new type oil absorption felt for potential application to ocean oil spill. Proced Environ Sci 10:849-853

Saleem J, Bazargan A, Barford J, McKay G (2014) Super-fast oil uptake using porous ultra-high molecular weight polyethylene sheets. Polym Adv Technol 25(10):1181-1185

Schmenk B, Miez-Meyer R, Steffens M, Wulfhorst B, Gleixner G (2000) Polypropylene fibre table. Chem Fibers Int 50(3):233-253

Sęk J, Shtyka OS, Szymczak K (2015) Modeling of the spontaneous polypropylene sorbents imbibition with emulsions. J Environ Eng Landsc 23(2):83-93

Srinivasan A, Viraraghavan T (2010) Oil removal from water using biomaterials. Bioresour Technol 101:6594-6600

Teas Ch, Kalligeros S, Zanikos F, Stournas S, Lois E, Anastopoulos G (2001) Investigation of the effectiveness of absorbent materials in oil spills clean up. Desalination 140(3):259-264

Wake H (2005) Oil refineries: a review of their ecological impacts on the aquatic environment. Estuar Coast Shelf Sci 62(1-2):131-140

Walker AH, Ducey DL, Gould JR, Nordvik AB (1993) Formation and breaking of water-in-oil emulsions. In: Workshop Proceedings. Marine Spill Response Corporation, DC

Wei QF, Mather RR, Fotheringham AF, Yang RD (2003) Evaluation of polypropylene nonwoven oil sorbents in marine oil spill recovery. Mar Pollut Bull 46(6):780-783

Wei QF, Mather RR, Fotheringham AF (2005) Oil removal from used sorbents using a biosurfactant. Bioresour Technol 96(3):331-334

Wu J, Wang N, Wang L, Dong H, Zhao Y, Jiang L (2012) Electrospun porous structure fibrous film with high oil adsorption capacity. ACS Appl Mater Interfaces 4(6):3207-3212

Xie H, Yapa PD, Nakata K (2007) Modeling emulsification after an oil spill in the sea. J Mar Syst 68(3-4):489-506

Xue HT, Fang ZN, Yang Y, Huang JP, Zhou LW (2006) Contact angle determined by spontaneous dynamic capillary rises with 
hydrostatic effects: experiment and theory. Chem Phys Lett 432(1-3):326-330

Zhang P, Wang L (2010) Extended Langmuir equation for correlating multilayer adsorption equilibrium data. Sep Purif Rev 70(3):367-371

Zhao HY, Li KW (2009) A fractal model of production by spontaneous water imbibition. Latin American and Caribbean Petroleum Engineering Conference held in Cartagena
Zhmud BV, Tiberg F, Hallstensson K (2000) Dynamics of capillary rise. J Coll Interface Sci 228:263-269

Zhu H, Qiu S, Jiang W, Wu D, Zhang C (2011) An evaluation of electrospun polyvinylchloride/polystyrene fibers as sorbent materials for oil spill cleanup. Environmental 45:4527-4531 